\title{
High-resolution observations of small-scale gravity waves and turbulence features in the $\mathrm{OH}$ airglow layer
}

\author{
René Sedlak $^{1}$, Patrick Hannawald ${ }^{2}$, Carsten Schmidt ${ }^{1}$, Sabine Wüst ${ }^{1}$, and Michael Bittner ${ }^{1,2}$ \\ ${ }^{1}$ German Remote Sensing Data Center, German Aerospace Center, Oberpfaffenhofen, Germany \\ ${ }^{2}$ Institute of Physics, University of Augsburg, Augsburg, Germany \\ Correspondence to: René Sedlak (rene.sedlak@dlr.de)
}

Received: 8 September 2016 - Published in Atmos. Meas. Tech. Discuss.: 12 September 2016

Revised: 9 November 2016 - Accepted: 25 November 2016 - Published: 12 December 2016

\begin{abstract}
A new version of the Fast Airglow Imager (FAIM) for the detection of atmospheric waves in the $\mathrm{OH}$ airglow layer has been set up at the German Remote Sensing Data Center (DFD) of the German Aerospace Center (DLR) at Oberpfaffenhofen $\left(48.09^{\circ} \mathrm{N}, 11.28^{\circ} \mathrm{E}\right)$, Germany. The spatial resolution of the instrument is $17 \mathrm{~m} \mathrm{pixel}^{-1}$ in zenith direction with a field of view $(\mathrm{FOV})$ of $11.1 \mathrm{~km} \times 9.0 \mathrm{~km}$ at the OH layer height of ca. $87 \mathrm{~km}$. Since November 2015, the system has been in operation in two different setups (zenith angles 46 and $0^{\circ}$ ) with a temporal resolution of 2.5 to $2.8 \mathrm{~s}$.

In a first case study we present observations of two small wave-like features that might be attributed to gravity wave instabilities. In order to spectrally analyse harmonic structures even on small spatial scales down to $550 \mathrm{~m}$ horizontal wavelength, we made use of the maximum entropy method (MEM) since this method exhibits an excellent wavelength resolution. MEM further allows analysing relatively short data series, which considerably helps to reduce problems such as stationarity of the underlying data series from a statistical point of view. We present an observation of the subsequent decay of well-organized wave fronts into eddies, which we tentatively interpret in terms of an indication for the onset of turbulence.

Another remarkable event which demonstrates the technical capabilities of the instrument was observed during the night of 4-5 April 2016. It reveals the disintegration of a rather homogenous brightness variation into several filaments moving in different directions and with different speeds. It resembles the formation of a vortex with a horizontal axis of rotation likely related to a vertical wind shear. This case shows a notable similarity to what is expected from theoretical modelling of Kelvin-Helmholtz instabilities (KHIs).
\end{abstract}

The comparatively high spatial resolution of the presented new version of the FAIM provides new insights into the structure of atmospheric wave instability and turbulent processes. Infrared imaging of wave dynamics on the subkilometre scale in the airglow layer supports the findings of theoretical simulations and modellings.

\section{Introduction}

The reaction of hydrogen and ozone produces molecular oxygen and vibrational-rotationally excited hydroxyl $(\mathrm{OH})^{*}$. $\mathrm{OH}^{*}$ relaxes by emitting electromagnetic radiation, especially in the SWIR (shortwave infrared, commonly defined from 0.9 to $1.7 \mu \mathrm{m}$ ) range (Bates and Nicolet, 1950). The hydroxyl forms a Chapman layer at the upper mesosphere and lower thermosphere and is the brightest component of the airglow phenomenon. The peak concentration of $\mathrm{OH}^{*}$ is located at an altitude of ca. $87 \mathrm{~km}$ with a half-width of about $8 \mathrm{~km}$ (Baker and Stair, 1988). The $\mathrm{OH}^{*}$ mean emission altitude exhibits low annual variability (e.g. von Savigny, 2015). Since the density of the atmosphere decreases exponentially with increasing height, the amplitudes of upward propagating atmospheric waves considerably grow due to the conservation of energy. Once the waves reach the airglow layer, they influence the intensity of the airglow emission due to temperature and density variations. This makes the airglow an established phenomenon for the investigation of atmospheric dynamics. Furthermore, based on temperature data derived from a Na lidar, Gardner et al. (2002) concluded that the vertical heat flux due to wave dissipation is maximum near the mesopause altitude region because of the reduced stability 
due to the large temperature lapse rate and the low buoyancy parameter below the mesopause. It is thus expected to observe gravity wave breaking processes in this altitude region.

Gravity waves are an intensively investigated feature within atmospheric dynamics. Their ability to transport energy and momentum over long distances through the atmosphere makes them an important factor to be considered in climate studies and meteorology. It is well known that gravity wave spectra extend over a few magnitudes of the wavelength scale. Horizontal wavelengths from a few kilometres up to about thousand kilometres are usually observed.

Energy and momentum transported by atmospheric waves are released into the surrounding atmosphere when instabilities lead to turbulence. Atmospheric instability can either be of dynamical or of convective nature. Dynamical instabilities are caused by wind shear, leading to formation of Kelvin-Helmholtz instabilities (KHIs; Browning, 1971), whereas convective instabilities are induced when the temperature lapse rate gets superadiabatic (Fritts and Alexander, 2003).

Turbulent processes are characterized to cover several magnitudes of the length scale. The complete range of turbulence can be divided into three parts: within the largest regime, the so-called buoyancy subrange, energy is still carried by waves and the buoyancy force is dominating viscosity. Distortions on scales below the buoyancy subrange let the transported energy cascade to smaller and even smaller structures (inertial subrange) where energy is still conserved within the eddies until it is dissipated into the atmosphere due to viscous damping (viscous subrange) (e.g. Lübken et al., 1987). It must be noted that the scale limits between the three subranges are strongly dependent on the altitude. Within the abovementioned region of the $\mathrm{OH}^{*}$ peak concentration at an altitude of about $87 \mathrm{~km}$, the inertial subrange of turbulence is on a scale of a few hundreds of metres, starting just below $1 \mathrm{~km}$ and reaches the viscous subrange at about $30 \mathrm{~m}$ (Hocking, 1985).

In order to deduce horizontal information of atmospheric waves, infrared camera systems have come up as a highly promising remote sensing technique (see e.g. Peterson and Kieffaber, 1973; Herse et al., 1989; Moreels et al., 2008) as they allow obtaining images of atmospheric waves. Gravity wave activity at airglow altitude of the entire night sky can be imaged using all-sky lenses (Taylor et al., 1997; Smith et al., 2009, and many others). Another application for airglow imagers is the investigation of small-scale gravity wave structures, such as acoustic-gravity waves (e.g. Nakamura et al., 1999) or instability features (Hecht et al., 2014). A high spatio-temporal resolution is required for this purpose, which is achieved at the expense of the aperture angles of the imager. However, theoretical calculations especially in the spatial regime below $1 \mathrm{~km}$ are hardly supported by experimental observations yet, since a better spatial resolution than $500 \mathrm{~m} \mathrm{pixel}^{-1}$ is needed to image structures on these scales (Hecht et al., 2014).
As reported by Hannawald et al. (2016), the infrared imaging system FAIM (Fast Airglow Imager) is currently being operated by the German Aerospace Center (DLR) in order to continuously observe gravity waves in the $\mathrm{OH}$ airglow each night with a temporal resolution of $0.5 \mathrm{~s}$. Obtaining a mean spatial resolution of $200 \mathrm{~m} \mathrm{pixel}^{-1}$, smallscale gravity wave structures with wavelengths down to $2 \mathrm{~km}$ have already been observed. Such instruments are currently operated at the Sonnblick Observatory $\left(47.05^{\circ} \mathrm{N}\right.$, $12.97^{\circ} \mathrm{E}$ ) in Austria (FAIM 1) as well as at Oberpfaffenhofen $\left(48.09^{\circ} \mathrm{N}, 11.28^{\circ} \mathrm{E}\right)$, Germany (FAIM 4$)$, for routine observations within the international Network for the Detection of Mesospheric Chance (NDMC, http://wdc.dlr.de/ndmc). A third system of this kind, FAIM 2, is installed for aircraft based observations on the DLR research aircraft FALCON.

In order to investigate even smaller-scale gravity wave signatures and turbulent features at mesopause heights, a further developed FAIM system, FAIM 3, is presented in this paper. Compared to earlier airglow imagers (Hecht et al., 1997; Yamada et al., 2001) the resolution has been improved by at least 1 order of magnitude in both space and time. Achieving a spatial resolution of $30 \mathrm{~m} \mathrm{pixel}^{-1}$ (zenith angle of $46^{\circ}$ ) and $17 \mathrm{~m} \mathrm{pixel}^{-1}$ (zenith angle of $0^{\circ}$ ) the entire inertial subrange as well as the beginning viscous subrange of turbulence at airglow altitude is accessible to this instrument. Additionally, the temporal resolution of no longer than $2.8 \mathrm{~s}$ allows investigating the development of transient processes like breaking wave fronts.

\section{Instrumentation}

FAIM 3 is the second version of the Fast Airglow Imagers established at DLR (Hannawald et al., 2016). It is based on the SWIR camera CHEETAH CL developed by Xenics nv. Its $640 \times 512$ pixel InGaAs sensor array with a pixel size of $20 \mu \mathrm{m} \times 20 \mu \mathrm{m}$ is sensitive to the spectral range from 0.9 to $1.7 \mu \mathrm{m}$. The raw signal is converted into a 14 bit digital stream, which is sent to a frame grabber via CameraLink. The sensor is thermoelectrically cooled down to an operational temperature of $233 \mathrm{~K}$. The dissipated heat is carried away by a closed circuit water cooling system. A schematic diagram of the entire setup is shown in Fig. 1.

All measurements presented here are performed at DLR at Oberpfaffenhofen $\left(48.09^{\circ} \mathrm{N}, 11.28^{\circ} \mathrm{E}\right)$, southern Germany. A $100 \mathrm{~mm}$ SWIR lens by Edmund Optics ${ }^{\circledR}$ is used. Its aperture angles have been determined to be $7.3^{\circ}$ in horizontal direction and $5.9^{\circ}$ in vertical direction. In the first measurement configuration the camera was mounted on a fixed stage with a zenith angle of $46^{\circ}$. The geometry of this arrangement implies a trapezium-shaped field of view (FOV) at the airglow layer, with a height of $18.6 \mathrm{~km}$ and a width of 15.2 to $16.9 \mathrm{~km}$ (see Hannawald et al., 2016, for details of the calculation). We note that the curvature of the Earth has been neglected within these considerations. Thus, horizontally an 


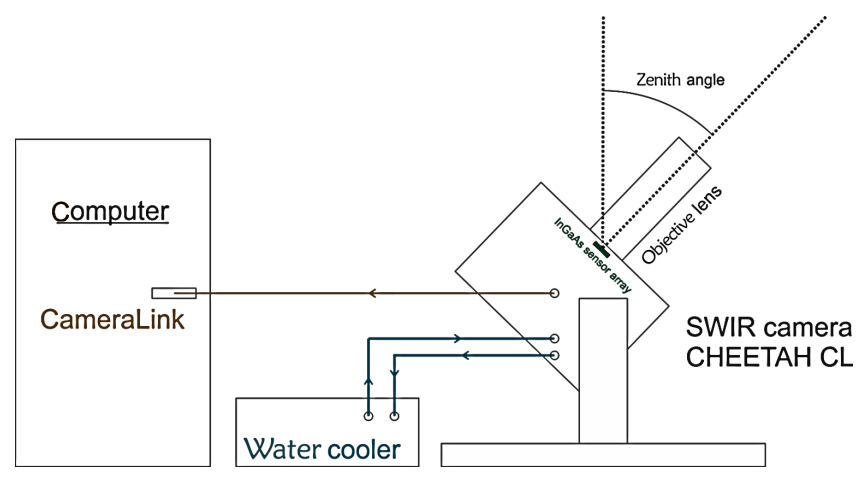

Figure 1. Schematic drawing of the FAIM 3 setup. The measurement configuration with a zenith angle of $46^{\circ}$ is shown here.

overall area of about $299 \mathrm{~km}^{2}$ is observed with a mean spatial resolution of $30 \mathrm{~m} \mathrm{pixel}^{-1}$. The camera is adjusted to an azimuth angle of $214^{\circ}$. In order to record a sufficiently strong airglow signal the integration time of the camera is set to $2.5 \mathrm{~s}$. The instrument in this configuration has been in operation from 18 to 30 November 2015. The data of the first case study were measured with this configuration.

In a second measurement configuration the FAIM 3 zenith angle is adjusted to $0^{\circ}$. Still using the $100 \mathrm{~mm}$ lens, a $11.1 \mathrm{~km} \times 9.0 \mathrm{~km}$ rectangular area $\left(100 \mathrm{~km}^{2}\right)$ of the airglow layer is acquired. This leads to a mean spatial resolution of $17 \mathrm{~m} \mathrm{pixel}^{-1}$. The integration time has to be raised to $2.8 \mathrm{~s}$ since the airglow intensity decreases with decreasing zenith angle due to the van Rhijn effect (van Rhijn, 1921).

A flat field correction is applied in order to remove the fixed pattern noise and the vignetting of the acquired images.

In order to better judge on the overall dynamical situation we operated the FAIM 3 zenith measurements simultaneously with the FAIM 4 instrument where FAIM 4 was allowed to sense the overall sky. The temporal resolution of FAIM 4 in this case was $1.5 \mathrm{~s}$ while the spatial resolution is roughly $400 \mathrm{~m} \mathrm{pixel}^{-1}$ within the image centre.

\section{Data analysis}

The horizontal wavelengths of gravity waves in an image can be estimated by analysing the data series of the pixel intensities along a cross section perpendicular to the wave fronts. The distortion resulting from the abovementioned trapeziumshaped FOV projected onto rectangular images has to be corrected prior to analysis so that an equidistant metric scale is provided. The images are mirrored and rotated into the proper azimuthal direction so that they can be rendered true to scale onto a map (see Fig. 2). This correction is performed using the same algorithm as described in Hannawald et al. (2016). As a typical example, Fig. 2a-f show a sequence of such images taken on 18 November 2015, between 22:59:55 and 23:04:11 UTC.
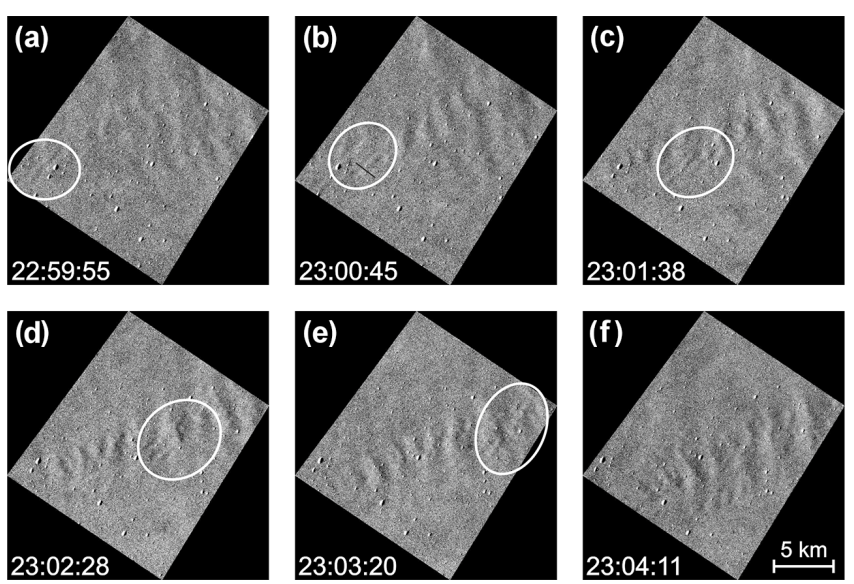

Figure 2. Image series of the case study of 18 November 2015 between 22:59:55 and 23:04:11 UTC. Difference images have been chosen for presentation purposes. The images are aligned to the north. The $1.7 \mathrm{~km}$ wave structure extends from the western to the eastern corner with several crests being arranged in a narrow corridor. The $550 \mathrm{~m}$ wave packet is framed in a white ellipse (a-e). One may perceive the beginning decay at 23:03:20 UTC. The entire sequence is shown in Video 1 of the Supplement. The black line, which is shown in (b), indicates the cross section along which the spectral analysis is performed in Figs. 3 and 4.

The periodic brightness variation related to a wavelike structure appears in the series of pixel intensities along a direction perpendicular to the wave fronts in the image, as shown in Fig. 3. Spectral analysis of the data series allows deducing the horizontal wavelengths. Since in our case the observed gravity waves are localized within a comparatively small area of the FOV and exhibit wavelengths of relatively small scales, the data series of pixel intensities extend over less than a hundred data points. Considering also the pronounced noise level, the maximum entropy method (MEM) turned out to be a powerful technique to estimate the spectral density of the data sets (Ulrych and Bishop, 1975), even if the data series is so short. As an example, Fig. 4 shows the MEM spectrum of the data series shown in Fig. 3.

The MEM provides an approach for estimating the spectral density of a time series with a high frequency resolution (e.g. Bittner et al., 1994). While other methods like the wavelet analysis try to fit a predefined function to the data, the MEM does not claim any assumptions about the theoretical shape of the signal but to be a stationary Gaussian process. According to Jaynes (1963) the most probable of all possible spectra describing a data set is the one which makes the fewest assumptions about the information the data contain. This corresponds to the state of maximum entropy. The MEM derives the most probable spectrum of a time series by fitting an autoregressive model (AR) of order $N$ to the data. The AR coefficients are determined using Burg's algorithm, which calculates them based on forward and backward predictions of the data. The major issue of the MEM is to find 


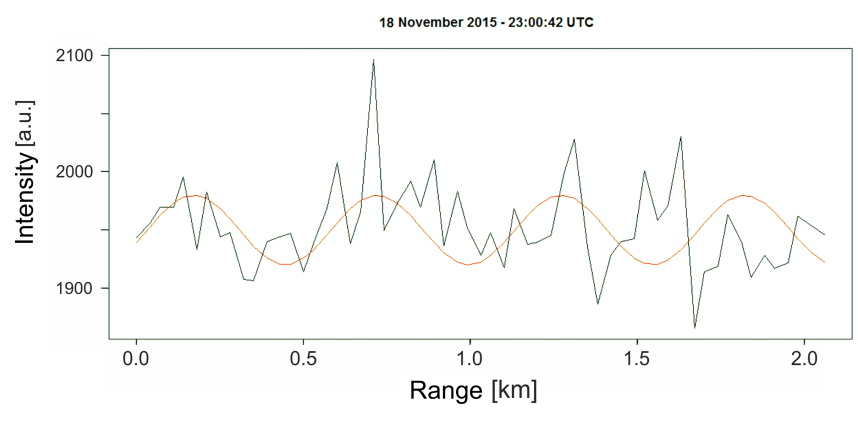

Figure 3. Measured intensities along a cross section perpendicular to the wavefronts (its position is indicated by the black line in Fig. 2b) in the image of 18 November 2015, 23:00:42 UTC. The pixel range has been converted into kilometres. The orange line shows an ideal $550 \mathrm{~m}$ wave (fitted by hand). Only the first half of the data series shows this signal, too.

the right order $N$ of the underlying AR process. If the order is chosen too small several frequency peaks may not be resolved, whereas an overestimated order will lead to further splitting of the peaks into spurious subpeaks due to noise approximation (e.g. Wüst and Bittner, 2006).

\section{Results}

During the night from 18 to 19 November 2015 observations were performed at a zenith angle of $46^{\circ}$, leading to a spatial resolution of approximately $30 \mathrm{~m} \mathrm{pixel}^{-1}$. In order to demonstrate the performance of the instrument, one interesting gravity wave event, registered between 22:59:25 and 23:04:33 UTC, is presented. The interval is shown in parts by a series of six images in Fig. 2a-f and in its entirety in Video 1 in the Supplement. First-order difference images (meaning the temporal derivation of the original signal, here $\Delta t=7.5 \mathrm{~s}$ ) are shown here to help better visualize wave structures. The difference of two images acts as a high-pass filter, suppressing long-period oscillations and highlighting the regions with varying intensity between the two images. However, the original data are used, of course, for spectral analysis in order to retrieve the correct spatial content. It is interesting to note that a smaller-scale wave packet is propagating from the left corner of the FOV (west) to the right corner (east), apparently advected by the background wind. The packet, which is marked by white circles in Fig. 2a-e, moves in the same direction as a second, larger wave structure but its wave fronts are tilted against each other horizontally by an angle of about $45^{\circ}$. The larger wave structure extends nearly over the entire image from the lower left to the upper right corner of Fig. 2a-f, and the quite limited horizontal width of the wave fronts makes the wave appear to be trapped in a narrow corridor in the airglow layer. Having nearly completely passed through the FOV the wave packet seems to decompose into some disordered features as time

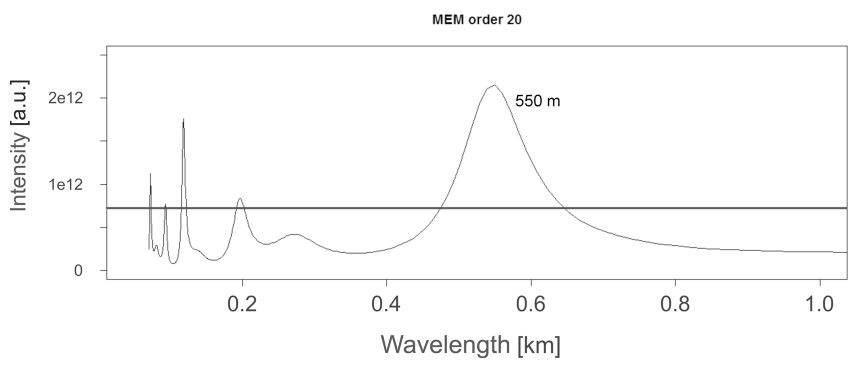

Figure 4. MEM spectrum (of the order of 20) of the data series shown in Fig. 3. The horizontal line marks the $99 \%$ level of significance. The peak of the $550 \mathrm{~m}$ wave packet is clearly recognizable.

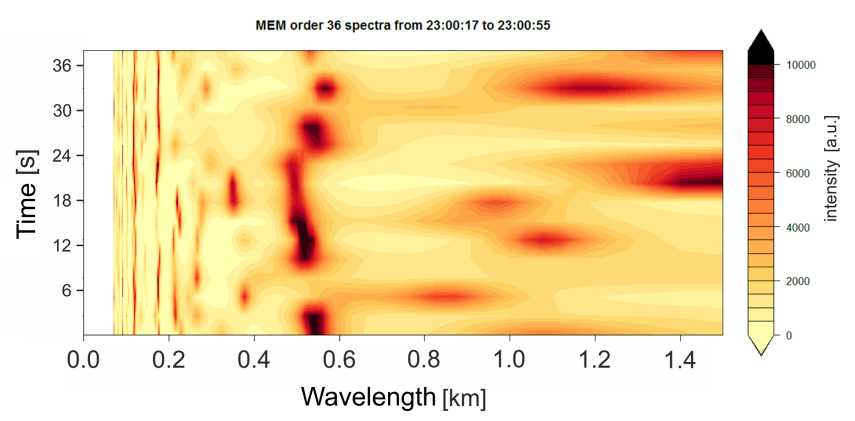

Figure 5. MEM spectra of the order of 36 of pixel intensities along the abovementioned cross section (its position is indicated by the black line in Fig. 2b) in every image taken between 23:00:17 and 23:00:55 UTC. The signature of the $550 \mathrm{~m}$ wave packet is found by the MEM nearly the entire period.

proceeds and is no longer visible in Fig. 2f. This is best recognizable at the end of Video 1 in the Supplement, starting at 23:03:20 UTC. Figure 3 shows in black the pixel intensities along a cross section perpendicular to the wave fronts of the small wave packet in the image acquired at 23:00:42 UTC (the position of the cross section is given in Fig. 2b). Visual inspection of the image series and the cross section shows that the wavelength of the wave packet is about $0.5 \mathrm{~km}$. The wavelength of the larger wave structure can be visually estimated as about $1.7 \mathrm{~km}$. For a more detailed analysis of the smaller structure, the MEM is applied to the data series of Fig. 3. An order of 20 is chosen as this corresponds to onethird of the number of data points, which has turned out to be a good choice for analysing short data series with fewer than 100 values (Bittner et al., 1994). The MEM spectrum in Fig. 4 exhibits a strong peak at about $550 \mathrm{~m}$. It is clearly above the $99 \%$ level of statistical significance and fits to the small-scale wave packet discovered in the images, as mentioned above. It is interesting to note that the MEM spectrum also reveals four other significant peaks at wavelengths well below $200 \mathrm{~m}$, which can also be found in the signal of Fig. 3 . In the following we will focus on the $550 \mathrm{~m}$ wave structure, as it is clearly recognizable in Video 1 in the Supplement. 


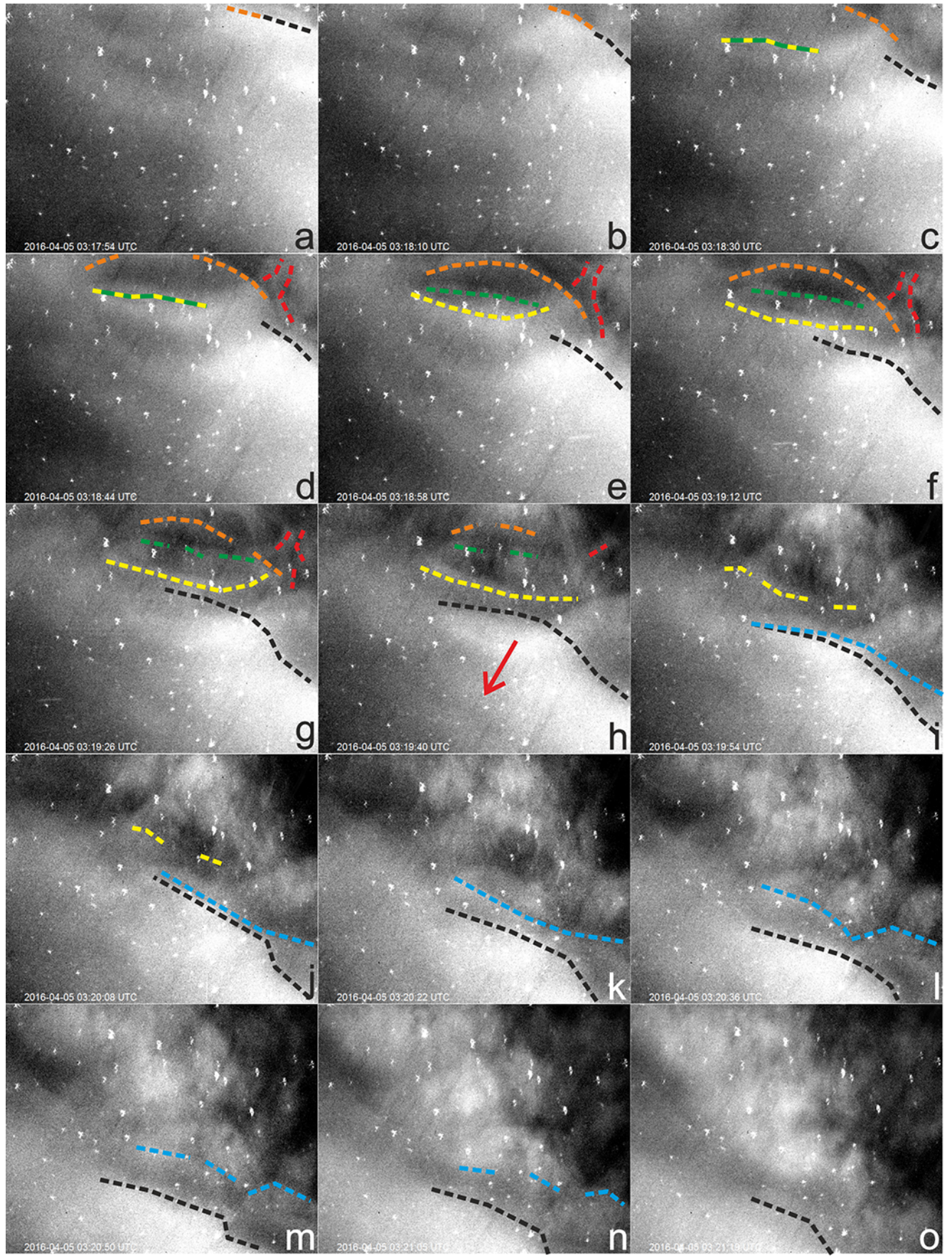

Figure 6. Image series of the second case study (5 April 2016 between 03:17:54 and 03:21:19 UTC). A wave front, indicated by the dashed black line, propagates into the direction the red arrow in (h) is indicating. While it continues propagating to the lower left, a filament separates from it on the left side (a, b, orange). At 03:18:30 UTC (c) a second filament structure becomes visible below the first filament. In the further course of the image series (e, f) it turns out to separate into two structures, one filament moving downward (yellow) and one stationary filament (green). At 03:19:26 UTC (g) the orange and the green structure begin to dissolve. The yellow structure continues propagating for a few more seconds and finally also starts decomposing at 03:19:54 UTC (i). At 03:18:44 UTC (d) two more filaments form at the upper right of the FOV right behind the initial wave front and are, in contrast to the other filaments, aligned perpendicular to it. They decompose at 03:19:26 UTC (g). While the dynamics of the filaments take their course and form a vortex, rotating around a horizontally oriented axis, the initial wave front (black) overtakes the other structures, retaining its original direction (indicated by the red arrow in $\mathbf{h}$ ). At about 03:19:54 UTC (i) another filament (blue) separates from it. This new filament remains stationary and starts decaying at 03:20:50 UTC (m). The wave front (black) keeps on propagating and leaves the FOV toward the lower left. The entire sequence is shown in Video 2 in the Supplement. 
It can be seen in the image series that the $550 \mathrm{~m}$ wave signal is visible in the examined cross section from 23:00:17 to 23:00:55 UTC. In a next step the same cross section as above is analysed for each image to derive some insight about the spatio-temporal evolution of the structure. Figure 5 shows the temporal development of the MEM spectrum. The order of the MEM has been raised to 36 ensuring that signatures of wavelengths below $1 \mathrm{~km}$ are fully resolved. A stable maximum in the spectrogram (Fig. 5) around a horizontal wavelength at about $550 \mathrm{~m}$ is obviously present over almost the entire time range.

Another interesting event was observed during the night from 4 to 5 April 2016. The images (see Fig. 6a-o) have been acquired at the zenith position with a spatial resolution of about $17 \mathrm{~m} \mathrm{pixel}^{-1}$. The dimensions of each image are approximately $11.1 \mathrm{~km} \times 9.0 \mathrm{~km}$. The zenith images show the sky as seen from the ground looking upwards. The upper image side corresponds to an azimuthal direction of $303^{\circ}$. Video 2 in the Supplement shows the entire event.

A wave front, indicated by the dashed black line in the images of Fig. 6, enters the FOV in the upper right corner. While it continues propagating to the lower left, a filament separates from it on the left side (Fig. 6a-b, orange). This filament moves more slowly than the wave front. At around 03:18:30 UTC (Fig. 6c) a second filament structure becomes visible below the first filament. In the further course of the image series (Fig. 6e-f) it turns out to separate into two structures, a filament moving downward (yellow) and a stationary filament (green). At about 03:19:26 UTC (Fig. 6g) the orange and the green structure begin to dissolve. The yellow structure continues propagating for a few more seconds and finally also starts decomposing at 03:19:54 UTC (Fig. 6i). At 03:18:44 UTC (Fig. 6d) two more filaments form at the upper right of the FOV right behind the initial wave front and are, in contrast to the other filaments, aligned perpendicular to it. They decompose at 03:19:26 UTC (Fig. 6g). While the dynamics of the filaments take their course and form a vortex, rotating around a horizontally oriented axis, the initial wave front (black) overtakes the other structures, retaining its original direction (indicated by the red arrow in Fig. 6h). At about 03:19:54 UTC (Fig. 6i) another filament (blue) separates from it. This new filament remains stationary and starts decaying at 03:20:50 UTC (Fig. 6m). The wave front (black) keeps on propagating and leaves the FOV toward the lower left.

To put the observations into a larger spatial context, the FAIM 3 data are compared to simultaneous all-sky measurements taken by the FAIM 4 instrument. Since the two cameras are deployed next to each other, the FOV of FAIM 3 is embedded in the centre of the FOV of FAIM 4. The FAIM 4 measurements are presented in Video 3 in the Supplement. Besides the normal image, the difference image (time difference of $60 \mathrm{~s}$ ) is displayed on the right side; both images are rotated northward. The approximate FOV of FAIM 3 is indicated by the white boxes in Video 3 in the Supplement.

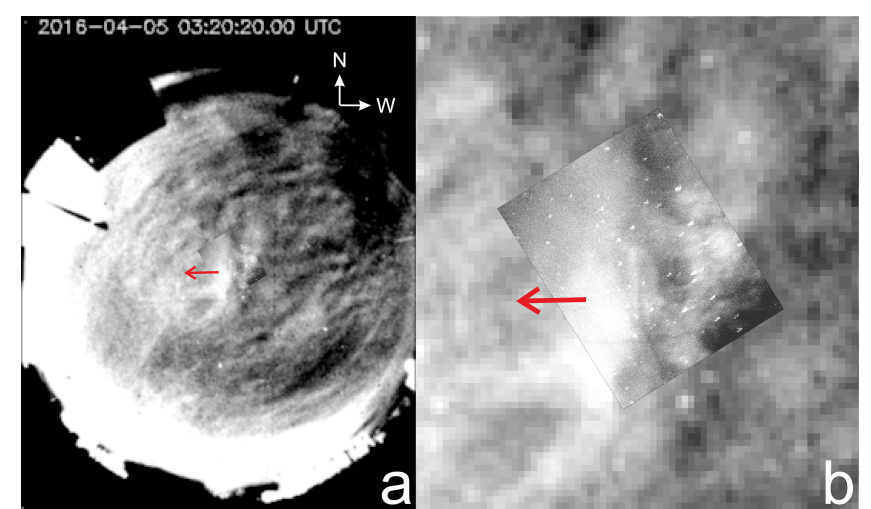

Figure 7. FAIM 4 all-sky image taken on 5 April 2016 at 03:20:20 UTC (a) and the magnified (zoom factor of 4) image centre (b). The entire sequence is shown in Video 3 in the Supplement. Due to their spatial structure and their wavelength, we interpret the patchy structures in the starry sky as gravity wave fronts in the airglow layer. Comparison with the respective FAIM 3 image, which has been placed at its correct position in the middle of the all-sky image, shows how the small-scale details of the wave crest can be resolved with the new instrument. The direction of propagation is indicated by the red arrow and matches with the observations of FAIM 3.

The all-sky images reveal a clear and starry sky with high gravity wave activity, which can be determined on the basis of the characteristic patchy structures. The remarkable structure observed by FAIM 3 can be found again in Video 3 in the Supplement as a bright feature within the white box, propagating to eastern direction, which agrees with the FAIM 3 observations. Figure 7 shows the FAIM 4 all-sky image at 03:20:20 UTC with the respective FAIM 3 image embedded into it (Fig. 7a) as well as the image centre magnified by a factor of 4 (Fig. 7b).

\section{Discussion}

The first of the two case studies presented here shows a wave packet with a horizontal wavelength of about $550 \mathrm{~m}$ moving in the same direction as a $1.7 \mathrm{~km}$ wave with the wave fronts being tilted about $45^{\circ}$ against the other structure. We interpret the wave packet as a ripple structure (see e.g. Peterson, 1979). Ripples are spatially limited wave structures with a short lifetime (Adams et al., 1988) and are caused by atmospheric instabilities (Taylor and Hapgood, 1990). As the $550 \mathrm{~m}$ wave packet does not form within the FOV, its lifetime cannot be estimated from our data. However, the appearance and disappearance of the $1.7 \mathrm{~km}$ wave is located within the FOV, which allows estimating its lifetime to about $16 \mathrm{~min}$. According to Taylor and Hapgood (1990) this feature can also be referred to as a ripple structure. The fact that the spatial extent of the wave fronts perpendicular to the direction motion is much smaller than the extent of the wave packet in 
propagation direction is unusual (ratio approximately $1: 6$ ). Obviously, it cannot be described as a plane wave (of infinite extent). It may rather be trapped in a duct, or the entire pattern, which appears to be a wave structure, may indeed be an instability feature itself, which is not unlikely given the small dimension with an apparent wavelength of $1.7 \mathrm{~km}$. It may be consequently speculated that the $1.7 \mathrm{~km}$ wave itself is a possible instability feature of a larger-scale wave with a horizontal wavelength of several kilometres and decays into the $550 \mathrm{~m}$ wave packet as a subordinate instability structure. Such dynamics have already been observed on larger scales in Hecht et al. (2014). Assuming the same scenario here, the ratio of primary to secondary wavelength is about 3.1. This number agrees quite well with earlier KHI models (Klaassen and Peltier, 1991).

While dynamical instability manifests as subordinate wave structures parallel to the initial wave fronts, convective instability emerges as wave structures perpendicular to the initial gravity wave (Andreassen et al., 1994; Fritts et al., 1994). Taylor and Hapgood (1990) assume ripples to be the signature of KHIs, thus being related to dynamical instability. However, past observations do not agree about this issue. While Yamada et al. (2001) have presented images of instability features aligned parallel to a breaking gravity wave, Hecht et al. (1997) have observed ripple structures that were aligned perpendicular to an initial wave, which rather assigns those ripples to be caused by convective instability. In our observations none of both cases is favoured as the wave fronts of the $550 \mathrm{~m}$ wave packet are aligned by an angle of $45^{\circ}$ to the superordinate $1.7 \mathrm{~km}$ wave. This supports the assumption of Hecht (2004) that some ripples may be generated by the combination of dynamical and convective instability. According to Fritts et al. (1996) an initially convectively driven instability structure can be rotated by the background wind shear. This could be a possible explanation for the tilt of the wave fronts.

At around 23:03:30 UTC the $550 \mathrm{~m}$ wave packet starts to collapse into some disordered features, which we tentatively assign to turbulence. Following the considerations of Hocking (1985), observations on that scale are already situated in the inertial subrange at airglow altitudes. Similar turbulentlooking features resulting of atmospheric instabilities have been found in the measurements of Hecht et al. (2014) in the buoyancy regime. Related direct numerical simulations (DNSs) (see Fritts et al., 2014) to that data have already predicted secondary instability features below $1 \mathrm{~km}$, which had not been able to observe with an airglow imager so far. However, it must be stressed that these interpretations remain speculative at this stage as the focus of this paper is to demonstrate the capability of the FAIM 3 instrument to resolve smaller-scale dynamics in the mesopause altitude region. Alternatively, the periodic structures could simply be remarkably small-scale gravity waves with wavelengths of $1.7 \mathrm{~km}$ and $550 \mathrm{~m}$ as well, without being results of atmospheric instability.
The second interesting event detected by FAIM 3 is a wave front, which partly separates into filament-like features while propagating through the FOV. Most of the filaments emerge parallel to the incident wave front and develop different velocities so that the impression of a horizontally rotating vortex arises. This can likely be assigned to instability driven by wind shear, i.e. KHIs. The filament structures decay into disorganized features, which resemble the turbulent collapse of the wave packet on 18 November 2015 . On larger scales, other airglow observations exhibit similarlooking breakdown events of gravity wave fronts, like in Hecht et al. (2014). The effects of KHI dynamics in the airglow layer have been modelled using DNSs and large eddy simulations (Fritts et al., 2014). The vortex structure as well as the filament features turns out to be a typical manifestation of turbulence due to KHIs. However, in our observations also some filaments aligned perpendicular to the initial wave front have been formed (Fig. 6d-g, red). These indicate the presence of convective instability. The high gravity wave activity of the overall night sky, revealed by FAIM 4 all-sky measurements at the same time, certainly contributes to atmospheric instability by influencing the lapse rate. Thus it could be again the combination of dynamical and convective instability which triggers the event on 5 April 2016.

The aforementioned wave front is also visible in the allsky images, but a close inspection of Video 3 in the Supplement hardly allows perceiving indications for the separation of parts from the bright crest. Zooming into the all-sky image (Fig. 7b) shows that only the high-resolution measurements of FAIM 3 can reveal closer details of this structure.

\section{Summary and conclusions}

In order to observe smaller-scale gravity wave events and instabilities or turbulence features in the metre regime, the established airglow imaging system FAIM (Hannawald et al., 2016) has been improved with regard to spatial resolution, using an InGaAs sensor array with the 4-fold number of pixels (327680) and a $100 \mathrm{~mm}$ SWIR lens manufactured by Edmund Optics ${ }^{\circledR}$. The mean spatial resolution of $200 \mathrm{mpixel}^{-1}$ at a $45^{\circ}$ zenith angle and $120 \mathrm{mpixel}^{-1}$ at zenith position achieved by the established FAIM system has been increased to 30 and $17 \mathrm{~m} \mathrm{pixel}^{-1}$ respectively. Measurements have been taken at Oberpfaffenhofen $\left(48.09^{\circ} \mathrm{N}\right.$, $11.28^{\circ} \mathrm{E}$ ), southern Germany, at a zenith angle of $46^{\circ}$ and an azimuth angle of $214^{\circ}$ as well as in zenith position.

Two case studies are presented. On 18 November 2015 from 22:59:25 to 23:04:33 UTC a wavelike structure with a wavelength of $1.7 \mathrm{~km}$ and a smaller feature with a wavelength of $550 \mathrm{~m}$ propagate in the same direction. Their wave fronts are tilted against each other by an angle of approximately $45^{\circ}$. The $1.7 \mathrm{~km}$ wave is estimated as having a lifetime of about $16 \mathrm{~min}$, which leads to the presumption that it might be an instability feature of a larger gravity wave with 
a wavelength of several kilometres, which cannot be seen in our small FOV. Following this hypothesis, the $550 \mathrm{~m}$ structure could be the resulting subsequent instability feature. Another possibility is that the $550 \mathrm{~m}$ wave packet could be the primary instability feature caused by a small $1.7 \mathrm{~km}$ gravity wave. Both cases agree with the theory of turbulence: when atmospheric instabilities lead to the breakdown of waves, their transported energy is cascaded to smaller-scale structures in the inertial subrange. These considerations combined with the ratio of the wavelengths, which fits to values of earlier theoretical studies like Klaassen and Peltier (1991), suggest that the event of 18 November 2015 could be triggered by KHIs.

Zenith measurements on 5 April 2016 from 03:15:58 to 03:27:46 UTC exhibit the breakdown of a wave front into a vortex structure and the subsequent decay into disorganized features, probably due to turbulence. Characteristic dynamics of filament-like features indicate that instability could be generated by wind shear. The observations look similar to modellings of KHI development and the consecutive turbulence dynamics of waves in the airglow layer (Fritts et al., 2014). Comparisons with parallel measurements of FAIM 4 obtaining all-sky images reveal the high gravity wave activity all over the sky at that time, which might have contributed to increased atmospheric instability.

It has been demonstrated that FAIM 3 is able to image the dynamics of gravity waves on scales significantly below $1 \mathrm{~km}$. FAIM 3 not only resolves the entire inertial subrange; it also provides insight into the beginning viscous subrange of turbulence. As concerns airglow imaging this opens a new scale range of dynamic processes that can be monitored, like shown in the first case study. Whereas structures like the larger one (periodicity $\sim 1.7 \mathrm{~km}$ ) can now be studied in greater detail with FAIM 3, structures like the smaller one $(550 \mathrm{~m})$ are now observable for the first time at all.

Concerning the connection of our observations with previous work in terms of scientific aspects, the second event is more evident. It shows the formation and temporal evolution of an instability feature. Due to the high temporal resolution $(2.8 \mathrm{~s})$ one can determine the initial formation of this structure and its later orientation relative to the initial wave field. Thus, observations of this kind are valuable for determining the nature of instability concerning the question of whether such features are primarily driven convectively or dynamically.

In this context several previous studies (e.g. Yamada et al., 2001; Hecht et al., 2004; Fritts et al., 1996) question of whether "ripples" were initially formed parallel or perpendicular to the gravity wave fronts and then rotated by the local wind fields or formed as a combination of both instabilities. These possibilities severely complicate scientific interpretation of ripple occurrence. With the new observation capabilities provided by the FAIM 3 we can now study this initial formation in greater detail. The two instability events presented in this paper appear to be driven dynamically, but in both cases there are also indications of the presence of convective instability, which suggests that these two instability mechanisms could actually accompany each other.

FAIM 3 observations support earlier simulations of instability dynamics as presented in Fritts et al. (2014). The typical vortex structures and the decay into eddies also appear in the respective airglow modelling. Like outlined there and in the companion experimental paper (Hecht et al., 2014), the simulations have predicted such small features that could not have been resolved by airglow imagers at that time. Apart from that, FAIM 3 is an ideal addition to all-sky imagers, since it provides the opportunity to more closely investigate specific airglow structures with a much higher spatial resolution, as demonstrated in the second case study in this paper.

Operational zenith measurements with a mean spatial resolution of $17 \mathrm{~m} \mathrm{pixel}^{-1}$ and a temporal resolution of $2.8 \mathrm{~s}$ have been performed automatically every night since 22 February 2016 at Oberpfaffenhofen, Germany.

\section{Data availability}

The data are archived at the WDC-RSAT (World Data Center for Remote Sensing of the Atmosphere, http://wdc.dlr.de). FAIM 3 is part of the Network for the Detection of Mesospheric Change, NDMC (http://wdc.dlr.de/ndmc).

\section{The Supplement related to this article is available online at doi:10.5194/amt-9-5955-2016-supplement.}

Acknowledgements. Parts of this research received funding from the Bavarian State Ministry of the Environment and Consumer Protection by grant number TUS01UFS-67093.

The article processing charges for this open-access publication were covered by a Research

Centre of the Helmholtz Association.

Edited by: L. Hoffmann

Reviewed by: two anonymous referees

\section{References}

Adams, G. W., Peterson, A. W., Brosnahan, J. W., and Neuschaefer, J. W.: Radar and optical observations of mesospheric wave activity during the lunar eclipse of 6 July 1982, J. Atmos. Terr. Phys., 50, 11-20, 1988.

Andreassen, Ø., Wasberg, C. E., Fritts, D. C., and Isler, J. R.: Gravity wave breaking in two and three dimensions 1 . Model description and comparison of two-dimensional evolutions, J. Geophys. Res., 99, 8095-8108, 1994. 
Baker, D. J. and Stair, A. T.: Rocket Measurements of the Altitude Distributions of the Hydroxyl Airglow, Phys. Scripta, 37, 611$622,1988$.

Bates, D. R. and Nicolet, M.: Atmospheric Hydrogen, Publ. Astron. Soc. Pac., 62, 106-110, 1950.

Bittner, M., Offermann, D., Bugaeva, I. V., Kokin, G. A., Koshelkov, J. P., Krivolutsky, A., Tarasenko, D. A., Gil-Ojeda, M., Hauchecorne, A., Lübken, F.-J., de la Morena, B. A., Mourier, A., Nakane, H., Oyama, K. I., Schmidlin, F. J., Soule, I., Thomas, L., and Tsuda, T.: Long period/large scale oscillations of temperature during the DYANA campaign, J. Atmos. Terr. Phys., 56, 1675-1700, 1994.

Browning, K. A.: Structure of the atmosphere in the vicinity of large-amplitude Kelvin-Helmholtz billows, Q. J. Roy. Meteor. Soc., 97, 283-299, 1971.

Fritts, D. C. and Alexander, M. J.: Gravity wave dynamics and effects in the middle atmosphere, Rev. Geophys., 41, 1003, doi:10.1029/2001RG000106, 2003.

Fritts, D. C., Isler, J. R., and Andreassen, Ø.: Gravity wave breaking in two and three dimensions 2 . Three-dimensional evolution and instability structure, J. Geophys. Res., 99, 8109-8123, 1994.

Fritts, D. C., Garten, J. F., and Andreassen, Ø.: Wave breaking and transition to turbulence in stratified shear flows, J. Atmos. Sci., 53, 1057-1085, 1996.

Fritts, D. C., Wan, K., Werne, J., Lund, T., and Hecht, J. H.: Modeling the implications of Kelvin-Helmholtz instabilty dynamics for airglow observations, J. Geophys. Res.-Atmos., 119, 88588871, doi:10.1002/2014JD021737, 2014.

Gardner, C. S., Zhao, Y., and Liu, A. Z.: Atmospheric stability and gravity wave dissipation in the mesopause region, J. Atmos. Sol.Terr. Phy., 64, 923-929, 2002.

Hannawald, P., Schmidt, C., Wüst, S., and Bittner, M.: A fast SWIR imager for observations of transient features in $\mathrm{OH}$ airglow, Atmos. Meas. Tech., 9, 1461-1472, doi:10.5194/amt-9-1461-2016, 2016.

Hecht, J. H.: Instability layers and airglow imaging, Rev. Geophys., 42, RG1001, doi:10.1029/2003RG000131, 2004.

Hecht, J. H., Walterscheid, R. L., Fritts, D. C., Isler, J. R., Senft, D. C., Gardner, C. S., and Franke, S. J.: Wave breaking signatures in $\mathrm{OH}$ airglow and sodium densities and temperatures 1. Airglow imaging, Na lidar, and MF radar observations, J. Geophys. Res., 102, 6655-6668, 1997.

Hecht, J. H., Wan, K., Gelinas, L. J., Fritts, D. C., Walterscheid, R. L., Rudy, R. J., Liu, A. Z., Franke, S. J., Vargas, F. A., Pautet, P. D., Taylor, M. J., and Swenson, G. R.: The life cycle of instability features measured from the Andes Lidar Observatory over Cerro Pachon on 24 March 2012, J. Geophys. Res. Atmos., 119, 88728898, 2014.

Herse, M., Thuillier, G., Camman, G., Chevassut, J.-L., and Fehrenbach, M.: Ground based instrument for observing near IR nightglow inhomogeneities at zenith and throughout the sky, Appl. Optics, 28, 3944-3949, doi:10.1364/AO.28.003944, 1989.

Hocking, W. K.: Measurement of turbulent energy dissipation rates in the middle atmosphere by radar techniques: A review, Radio Sci., 20, 1403-1422, 1985.
Jaynes, E. T.: New engineering applications of information theory, Proceedings of the first symposium on engineering applications of random function theory and probability, edited by: Bogdanoff, J. L. and Kozin, F., John Wiley, New York, 1963.

Klaassen, G. P. and Peltier, W. R.: The influence of stratification on secondary instability in free shear layers, J. Fluid Mech., 227, 71-106, 1991.

Lübken, F.-J., von Zahn, U., Thrane, E. V., Blix, T., Kokin, G. A., and Pachomov, S. V.: In situ measurements of turbulent energy dissipation rates and eddy diffusion coefficients during MAP/WINE, J. Atmos. Terr. Phys., 49, 763-775, 1987.

Moreels, G., Clairemidi, J., Faivre, M., Mougin-Sisini, D., Kouahla, M. N., Meriwether, J. W., Lehmacher, G. A., Vidal, E., and Veliz, O.: Stereoscopic imaging of the hydroxyl emissive layer at low latitudes, Planet. Space Sci., 56, 1467-1479, 2008.

Nakamura, T., Higashikawa, A., Tsuda, T., and Matsuhita, Y.: Seasonal variations of gravity wave structures in $\mathrm{OH}$ airglow with a CCD imager at Shigaraki, Earth Planets Space, 51, 897-906, 1999.

Peterson, A. W.: Airglow events visible to the naked eye, Appl. Optics, 18, 3390-3393, doi:10.1364/AO.18.003390, 1979.

Peterson, A. W. and Kieffaber, L. M.: Infrared Photography of $\mathrm{OH}$ Airglow Structures, Nature, 242, 321-322, 1973.

Smith, S., Baumgardner, J., and Mendillo, M.: Evidence of mesospheric gravity-waves generated by orographic forcing in the troposphere, Geophys. Res. Lett., 36, doi:10.1029/2008GL036936, 2009.

Taylor, M. J. and Hapgood, M. A.: On the origin of ripple-type wave structure in the $\mathrm{OH}$ nightglow emission, Planet. Space Sci., 38, 1421-1430, 1990.

Taylor, M. J., Pendleton Jr., W. R., Clark, S., Takahashi, H., Gobbi, D., and Goldberg, R. A.: Image measurements of short-period gravity waves at equatorial latitudes, J. Geogr. Res., 102, 26283 26299, doi:10.1029/96JD03515, 1997.

Ulrych, T. J. and Bishop, T. N.: Maximum entropy spectral analysis and autoregressive decomposition, Rev. Geophys. Space Phys., 13, 183-200, 1975.

van Rhijn, P. J.: On the brightness of the sky at night and the total amount of starlight, Publications of the Astronomical Laboratory at Groningen, 31, 1-83, 1921.

von Savigny, C.: Variability of $\mathrm{OH}(3-1)$ emission altitude from 2003 to 2011: Long-term stability and universality of the emission rate-altitude relationship, J. Atmos. Sol.-Terr. Phy., 127, 120 128, doi:10.1016/j.jastp.2015.02.001, 2015.

Wüst, S. and Bittner, M.: Non-linear resonant wave-wave interaction (triad): Case studies based on rocket data and first application to satellite data, J. Atmos. Sol.-Terr. Phy., 68, 959-976, 2006.

Yamada, Y., Fukunishi, H., Nakamura, T., and Tsuda, T.: Breaking of small-scale gravity waves and transition to turbulence observed in OH airglow, Geophys. Res. Lett., 28, 2153-2156, 2001. 